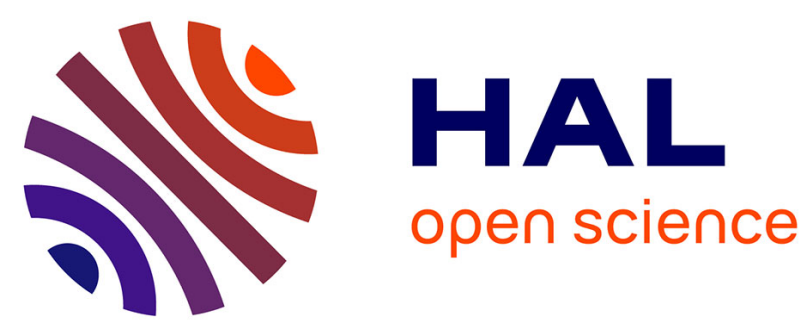

\title{
Susceptibility to five antifungals of strains isolated from chronically colonised cystic fibrosis patients receiving azole therapy
}

\author{
Adelina Amorim, Luísa Guedes-Vaz, Ricardo Araujo
}

\section{To cite this version:}

Adelina Amorim, Luísa Guedes-Vaz, Ricardo Araujo. Susceptibility to five antifungals of strains isolated from chronically colonised cystic fibrosis patients receiving azole therapy. International Journal of Antimicrobial Agents, 2010, 35 (4), pp.396. 10.1016/j.ijantimicag.2009.12.007 . hal-00567283

\section{HAL Id: hal-00567283 \\ https://hal.science/hal-00567283}

Submitted on 20 Feb 2011

HAL is a multi-disciplinary open access archive for the deposit and dissemination of scientific research documents, whether they are published or not. The documents may come from teaching and research institutions in France or abroad, or from public or private research centers.
L'archive ouverte pluridisciplinaire HAL, est destinée au dépôt et à la diffusion de documents scientifiques de niveau recherche, publiés ou non, émanant des établissements d'enseignement et de recherche français ou étrangers, des laboratoires publics ou privés. 


\section{Accepted Manuscript}

Title: Susceptibility to five antifungals of Aspergillus fumigatus strains isolated from chronically colonised cystic fibrosis patients receiving azole therapy

Authors: Adelina Amorim, Luísa Guedes-Vaz, Ricardo Araujo

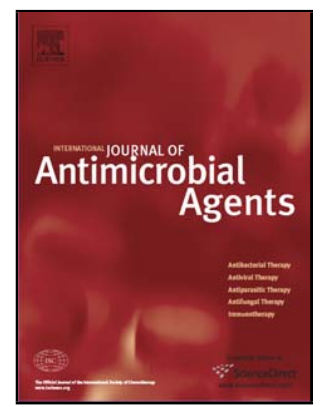

PII:

S0924-8579(10)00004-X

DOI:

doi:10.1016/j.ijantimicag.2009.12.007

Reference:

ANTAGE 3207

To appear in: International Journal of Antimicrobial Agents

Received date: $\quad 24-8-2009$

Revised date: $\quad 1-12-2009$

Accepted date: $\quad$ 4-12-2009

Please cite this article as: Amorim A, Guedes-Vaz L, Araujo R, Susceptibility to five antifungals of Aspergillus fumigatus strains isolated from chronically colonised cystic fibrosis patients receiving azole therapy, International Journal of Antimicrobial Agents (2008), doi:10.1016/j.ijantimicag.2009.12.007

This is a PDF file of an unedited manuscript that has been accepted for publication. As a service to our customers we are providing this early version of the manuscript. The manuscript will undergo copyediting, typesetting, and review of the resulting proof before it is published in its final form. Please note that during the production process errors may be discovered which could affect the content, and all legal disclaimers that apply to the journal pertain. 


\section{Susceptibility to five antifungals of Aspergillus fumigatus strains isolated from chronically colonised cystic fibrosis patients receiving azole therapy}

Adelina Amorim ${ }^{\mathrm{a}, \mathrm{b}}$, Luísa Guedes-Vaz ${ }^{\mathrm{c}}$, Ricardo Araujo ${ }^{\mathrm{d}, \mathrm{e}, \star}$

a Department of Pneumology, Faculty of Medicine, University of Porto, Alameda Prof. Hernani Monteiro, 4200-319 Porto, Portugal

${ }^{\mathrm{b}}$ Department of Pneumology, Hospital S. João, Alameda Prof. Hernani Monteiro, 4200319 Porto, Portugal

${ }^{c}$ Department of Paediatrics, Hospital S. João, Alameda Prof. Hernani Monteiro, 4200319 Porto, Portugal

d IPATIMUP, Institute of Pathology and Molecular Immunology, University of Porto, Rua Dr Roberto Frias, s/n, 4200-465 Porto, Portugal

${ }^{\text {e }}$ Department of Microbiology, Faculty of Medicine, University of Porto, Alameda Prof. Hernani Monteiro, 4200-319 Porto, Portugal

\section{ARTICLE INFO}

Article history:

Received 24 August 2009

Accepted 4 December 2009 
Keywords:

ABPA

Aspergillus fumigatus

Azole therapy

Cystic fibrosis

Microsatellite genotyping

Susceptibility testing

* Corresponding author. Tel.: +351 22557 0700; fax: +351 225570799.

E-mail address: ricjparaujo@yahoo.com (R. Araujo). 


\section{ABSTRACT}

Exposure of Aspergillus fumigatus to stressful antifungal therapies may result in decreased susceptibility. The aim of the present work was to evaluate the susceptibility to azole and non-azole antifungals of 159 isolates of $A$. fumigatus collected from cystic fibrosis (CF) patients receiving azole antifungal therapy. The genetic diversity of the fungal isolates was assessed using microsatellite genotyping, and some strains were found in patient's sputum samples more than 4 years apart. No resistant isolates [minimal inhibitory concentration (MIC)/minimal effective concentration (MEC) $\geq 4$ $\mu \mathrm{g} / \mathrm{mL}$ ] were identified to the antifungals amphotericin $\mathrm{B}$, caspofungin, itraconazole and voriconazole. A single A. fumigatus isolate was identified outside of the epidemiological cut-off of $0.25 \mu \mathrm{g} / \mathrm{mL}$ for posaconazole. Susceptibility of the recurrent isolates was in agreement with the susceptibility of the first isolate identified (100\% essential agreement). Even after azole exposure, several recurrent $A$. fumigatus strains were detected in the subsequent sputum samples. Development of resistance in A. fumigatus to antifungals appears to be rare among CF patients. However, it remains crucial to evaluate the importance of antifungal agents for allergic fungal diseases. 


\section{Introduction}

Cystic fibrosis (CF) patients are continuously exposed to microorganisms that may colonise their lungs, reducing respiratory function and enhancing pulmonary problems. Allergic bronchopulmonary aspergillosis (ABPA) is an intense allergic reaction, mainly in response to the presence of Aspergillus fumigatus, which can affect up to $15 \%$ of CF patients [1]. This allergic disease is normally treated with prescription of oral corticosteroid therapy and in a few critical clinical cases azole antifungals may be used $[1,2]$.

Aspergillus fumigatus is one of the most frequently isolated fungi from the lungs of $\mathrm{CF}$ patients [1]. It is commonly susceptible to the commercially available antifungals such as amphotericin B, caspofungin, itraconazole, voriconazole and posaconazole [3]. It was recently showed that CF patients may be chronically colonised with the same $A$. fumigatus strain over several years [4,5]. Exposure of $A$. fumigatus strains to distinct conditions and stressful therapies over long periods may sporadically lead to alterations in their susceptibility and to treatment failure $[6,7]$. The objective of the present work was to evaluate the susceptibility to azole and non-azole antifungals of 159 isolates of A. fumigatus collected from 11 patients, some of whom had been treated with azole antifungal therapy. 


\section{Materials and methods}

\subsection{Patients}

A group of 11 patients admitted to Hospital S. João (Porto, Portugal) and diagnosed with CF were studied. The diagnosis of CF was confirmed by screening CFTR mutations and positive evaluation of the sweat test. Patient age ranged from 15 years to 51 years. Patients were selected for this study following confirmation of chronic colonisation with $A$. fumigatus (more than three sputum samples with $A$. fumigatus in a previous year). Sputum samples were then collected from the patients between January 2005 and May 2009 (with a minimum of two samples from each patient at different times). Seven patients never received antifungal treatment, whilst four of the patients (patients $B, C, D$ and $E$ shown in Table 1) received azole antifungal therapy in addition to the recommended antibacterial treatment and oral corticosteroid therapy. Four of the studied patients (patients A, D, E and K shown in Table 1) matched with the modified criteria for diagnosis of ABPA in CF patients [2].

\subsection{Antifungal therapy}

Patient B received itraconazole between December 2007 and April 2008 (5 months) at a daily dose of $200 \mathrm{mg}$ due to a serious decrease of respiratory function and chronic colonisation with A. fumigatus and Scedosporium apiospermum. In April 2008, an infection by tuberculosis mycobacteria was diagnosed and treated and itraconazole therapy was suspended to avoid liver dysfunction. Because the decrease in respiratory 
function persisted, patient B started voriconazole therapy in June 2009 (200 mg/12 h). The patient is currently stabilised and remains on voriconazole therapy.

Patient C received itraconazole between November 2006 and September 2007 (12 months) at a daily dose of $400 \mathrm{mg}$ due to a decrease of respiratory function and clinical and radiological deterioration (mycetoma). The patient was chronically colonised with $A$. fumigatus and S. apiospermum. The clinical picture persisted after 1 year of itraconazole and in September 2007 the patient started voriconazole at $200 \mathrm{mg} / 12 \mathrm{~h}$. Sputum samples showed the absence of fungal colonisation following voriconazole treatment. Between January and March 2008 the patient developed hepatitis and voriconazole therapy was suspended. Patient $\mathrm{C}$ re-started voriconazole therapy in April 2008 without any further complication and remains on the antifungal treatment until now (August 2009).

Patient $D$ received voriconazole therapy for 12 months (from September 2007 to September 2008) at a dose of $200 \mathrm{mg} / 12 \mathrm{~h}$ due to serious haemoptysis and diagnosis of ABPA. The patient recovered and is currently stabilised.

Patient E received voriconazole therapy for 8 months (between June 2006 and February 2007 ; dose of $200 \mathrm{mg} / 12 \mathrm{~h}$ ) due to ABPA, with a favourable outcome. Presently (August 2009), the patient is receiving itraconazole therapy (daily dose of 200 mg initiated in September 2008) due to a serious haemoptysis and no criteria for ABPA. The patient is presently stabilised. 


\subsection{Fungal organisms}

Sputum samples were cultured in Sabouraud agar for 10 days at $30^{\circ} \mathrm{C}$ and $37^{\circ} \mathrm{C}$. A total of 159 isolates of $A$. fumigatus were recovered from the clinical samples. The isolates were identified based upon macroscopic and microscopic morphological characteristics following standard mycological procedures. Two A. fumigatus reference strains (ATCC 46645 and MYA 772) were included in the study. Prior to DNA isolation, moulds were grown for 5 days on Sabouraud dextrose agar slants (Difco, Detroit, MI) at $30{ }^{\circ} \mathrm{C}$. A sodium hydroxide-based method was used to extract DNA from conidia, as previously described [8]. DNA was suspended in $50 \mu \mathrm{L}$ of sterile water and stored at $20^{\circ} \mathrm{C}$.

\subsection{Antifungal susceptibility testing}

Determination of minimal inhibitory concentrations (MICs) of amphotericin B (BristolMyers SP, Dublin, Ireland), caspofungin (Merck Sharp \& Dohme, Whitehouse Station, $\mathrm{NJ}$ ), itraconazole (Janssen-Cilag, High Wycombe, UK), voriconazole (Pfizer Inc., New York, NY) and posaconazole (Schering-Plough Farma, Cacém, Portugal) was performed according to the Clinical and Laboratory Standards Institute broth microdilution method [9]. MICs were defined as the absence of visible growth after $48 \mathrm{~h}$ in the presence of the antifungals amphotericin B, itraconazole, voriconazole and posaconazole. For caspofungin, the minimal effective concentration (MEC) was 
determined as previously recommended [3]. Quality control strains Candida parapsilosis ATCC 22019 and Candida krusei ATCC 6258 were included each time testing was performed and antifungal MICs were within the expected limits $[10,11]$. Fungal strains were classified accordingly to the recently proposed in vitro breakpoints for amphotericin B, caspofungin, itraconazole and voriconazole (susceptible, MIC $<1$ $\mu \mathrm{g} / \mathrm{mL}$; intermediate, $\mathrm{MIC}=2 \mu \mathrm{g} / \mathrm{mL}$; and resistant, $\mathrm{MIC}>4 \mu \mathrm{g} / \mathrm{mL}$ ) [12]. For posaconazole, the fungal strains were classified according to recently proposed epidemiological cut-offs (wild-type, MIC $<0.25 \mu \mathrm{g} / \mathrm{mL}$; non-wild-type, MIC $>0.5 \mu \mathrm{g} / \mathrm{mL}$ ) [13]. Next, MICs of isolates with the same genotype were compared and were considered in essential agreement when the differences were no more than three dilutions (e.g. 0.5, 1.0 and $2.0 \mu \mathrm{g} / \mathrm{mL}$ ).

\subsection{Aspergillus fumigatus genotyping}

Microsatellite multiplex polymerase chain reaction (PCR) was performed using previously selected primers allowing the identification of microsatellites based on trinucleotide, tetranucleotide and pentanucleotide motifs located on different chromosomes [8]. Multiplex PCR was performed using $1 \mu \mathrm{L}$ of genomic DNA (1-5 $\mathrm{ng} / \mu \mathrm{L}$ ), $2.5 \mu \mathrm{L}$ of Multiplex PCR Master Mix (Qiagen, Hilden, Germany) and $0.5 \mu \mathrm{L}$ of a mix with eight primer pairs (final concentration of each primer $2 \mu \mathrm{M}$ ) in a final volume of $5 \mu \mathrm{L}$. The conditions for PCR amplification and sequencing reactions were as previously described [8]. 


\subsection{Statistical analysis}

Data were compared at a significance level of 0.05 by analysis of variance (ANOVA) using the Bonferroni correction, and Student's t-test for paired samples. Genotype diversity was calculated by Simpson's index of diversity. Statistical analysis for pair-wise linkage disequilibrium was performed by running the statistical software package Arlequin 3.1 (http://cmpg.unibe.ch/software/arlequin3/).

\section{Results}

Among the entire $A$. fumigatus collection, no resistant isolates (MIC/MEC $\geq 4 \mu \mathrm{g} / \mathrm{mL}$ ) were identified to the antifungals amphotericin $\mathrm{B}$, caspofungin, itraconazole and voriconazole (Table 1). The susceptibility of a few isolates was classified as intermediate to amphotericin B ( 3 isolates) and itraconazole (18 isolates), and a single A. fumigatus isolate was identified outside of the epidemiological cut-off of $0.25 \mu \mathrm{g} / \mathrm{mL}$ for posaconazole (this last isolate showed a susceptibility of $0.5 \mu \mathrm{g} / \mathrm{mL}$ for amphotericin B, $0.06 \mu \mathrm{g} / \mathrm{mL}$ for caspofungin, $2 \mu \mathrm{g} / \mathrm{mL}$ for itraconazole and $0.5 \mu \mathrm{g} / \mathrm{mL}$ for voriconazole). The number of isolates with intermediate susceptibility to itraconazole collected from patients was similar before and after azole treatment $(P>0.05)$.

The genotype diversity of $A$. fumigatus found in each patient's sample collection ranged from 0.6 to 1 , with an overall diversity of 0.92 when considering the entire group of 159 isolates. There was no significant relationship between $A$. fumigatus genotype diversity and the patient's diagnosis of ABPA $(P>0.05)$. Some strains were frequently found in 
the sputum samples of patients, occasionally more than 4 years apart. A total of 15 strains were repeatedly recovered from the clinical samples (total of 134 isolates) and the susceptibility of the repeated isolates (with the same genotype for all eight microsatellite markers) was in agreement with the susceptibility of the isolate that was first identified (100\% essential agreement). Eight of the recurrent (or chronic) strains of A. fumigatus had been exposed to an azole antifungal. Even after azole exposure, some recurrent $A$. fumigatus (four strains) were still detected in the subsequent sputum samples collected from the patients. Patient B was colonised with three of these recurrent strains and no significant differences were found between the susceptibility of the strains before and after exposure to itraconazole and voriconazole $(P>0.05)$.

Microvariation events were common among the clinical $A$. fumigatus isolates collected from six of the patients included in the study; three patients were diagnosed with ABPA. These events were more common between isolates recovered from the same sputum sample (e.g. events 4, 5 or 8), but they could be found in two samples from the same patient collected with more than 3 years apart.

\section{Discussion}

Oral corticosteroid therapy remains the first therapy for ABPA and there is still no consensus regarding how, how long or when antifungal drugs should be employed for treatment of patients with ABPA or those colonised with Aspergillus spp. [2,14]. Patients included in this study received azole antifungal therapy due to serious haemoptysis 
and/or decrease of respiratory function and clinical deterioration following administration of antibacterial treatment and/or corticosteroid therapy. In all cases there was verified clinical progress and favourable outcome. Antifungals have been seldom used for therapy among CF patients, but the long-standing and significant adverse effects of corticosteroid therapy, the difficulties associated with a firm diagnosis of ABPA and the clinical deterioration of chronically ill patients may result in the near future in an increase of antifungal usage among CF patients [14].

During the last decade there has been an increase, although infrequent, in the description of itraconazole resistance among A. fumigatus isolates obtained from patients with invasive fungal infections receiving antifungal drugs [6,13]. Resistance to voriconazole or posaconazole has been more rarely reported. Recently, the association of $A$. fumigatus azole resistance in allergic diseases was described resulting from prior exposure to an antifungal azole agent [7]. In all cases, treatment failure was reported and several $A$. fumigatus strains were identified as resistant to itraconazole, voriconazole and/or posaconazole. Conversely, in this study we could not detect any resistant $A$. fumigatus isolate to the five tested antifungals, azoles or non-azoles (a few fungal isolates were intermediate to amphotericin B or itraconazole). Nevertheless, we did not find unfavourable outcomes among the 11 evaluated patients.

Microsatellite genotyping results demonstrated that cystic CF fibrosis patients may be chronically colonised with the same strains, sometimes over several years as previously described $[4,5]$. In any case, exposure to an antifungal azole did not reduce the 
susceptibility of $A$. fumigatus genotypes chronically recovered from patients and, occasionally, among isolates that showed microvariation (disparity in a single marker). However, we can question whether the use of azole antifungal therapy was really effective. One-half of the recurrent $A$. fumigatus strains were detected in the subsequent sputum samples collected from patients. The limited number of studied patients and, in particular, the fact that all patients showed a good response to the therapy may limit additional conclusions. Nevertheless, we can assume with the present results that azole antifungal resistance is not a common event among $A$. fumigatus isolates collected from CF patients. Aspergillus fumigatus isolates may become resistant to antifungals only under particular conditions, uncommon therapeutic options and/or unusual structures. An example is mould biofilm that may be present in the CF lung and has already been described as exhibiting decreased antifungal drug susceptibility and longer viability compared with planktonic and sessile forms [15].

At present, it is crucial to re-evaluate several clinical therapies in order to avoid the appearance and dissemination of resistant strains. Azole antifungals remain the single oral solution available for aspergillosis and the development of resistance in these fungi seems to be rare. None the less, several strains can persist in the lungs of CF patients even after administration of azole antifungal therapy, questioning the significance of such treatment. It remains essential to define the clinical situations that indeed require this therapeutic alternative. Additionally, we also need to evaluate the importance of antifungal agents for allergic diseases in situations of failure of bacterial and corticosteroid treatments. 


\section{Acknowledgments}

The authors are grateful to Maria Luz Dias and Isabel Santos for excellent technical assistance.

\section{Funding}

This work was supported by grants from Fundação Calouste Gulbenkian (no. 35-9924S/2009), University of Porto and Santander Totta. RA was co-financed by the European Social Fund. IPATIMUP is partially supported by Fundação para a Ciência e Tecnologia (FCT), Programa Operacional Ciência e Inovação (POCl) and Programa Operacional do Potencial Humano (POPH - QREN).

\section{Competing interests}

None declared.

\section{Ethical approval}

Not required. 


\section{References}

[1] O'Sullivan BP, Freedman SD. Cystic fibrosis. Lancet 2009;373:1891-904.

[2] Stevens DA, Moss RB, Kurup VP, Knutsen AP, Greenberger P, Judson MA, et al. Allergic bronchopulmonary aspergillosis in cystic fibrosis—state of the art: Cystic Fibrosis Foundation Consensus Conference. Clin Infect Dis 2003;37(Suppl 3):S22564.

[3] Araujo R, Pina-Vaz C, Rodrigues AG. Susceptibility of environmental versus clinical strains of pathogenic Aspergillus. Int J Antimicrob Agents 2007;29:108-11.

[4] Vanhee LM, Symoens F, Bouchara JP, Nelis HJ, Coenye T. High-resolution genotyping of Aspergillus fumigatus isolates recovered from chronically colonised patients with cystic fibrosis. Eur J Clin Microbiol Infect Dis 2008;27:1005-7.

[5] de Valk HA, Klaassen CH, Yntema JB, Hebestreit A, Seidler M, Haase G, et al. Molecular typing and colonization patterns of Aspergillus fumigatus in patients with cystic fibrosis. J Cyst Fibros 2009;8:110-4.

[6] Snelders E, van der Lee HA, Kuijpers J, Rijs AJ, Varga J, Samson RA, et al. Emergence of azole resistance in Aspergillus fumigatus and spread of a single resistance mechanism. PLoS Med 2008;5:e219.

[7] Howard SJ, Cerar D, Anderson MJ, Albarrag A, Fisher MC, Pasqualotto AC, et al. Frequency and evolution of azole resistance in Aspergillus fumigatus associated with treatment failure. Emerg Infect Dis 2009;15:1068-76. 
[8] Araujo R, Pina-Vaz C, Rodrigues AG, Amorim A, Gusmão L. Simple and highly discriminatory microsatellite-based multiplex PCR for Aspergillus fumigatus strain typing. Clin Microbiol Infect 2009;15:260-6.

[9] Clinical and Laboratory Standards Institute. Reference method for broth dilution antifungal susceptibility testing of filamentous fungi; approved standard. 2nd ed. Document M38-A2. Wayne, PA: CLSI; 2008.

[10] Barry AL, Pfaller MA, Brown SD, Espinel-Ingroff A, Ghannoum MA, Knapp C, et al. Quality control limits for broth microdilution susceptibility tests of ten antifungal agents. J Clin Microbiol 2000;38:3457-9.

[11] Chaturvedi V, Ramani R, Ghannoum MA, Killian SB, Holliday N, Knapp C, et al. Multilaboratory testing of antifungal combinations against a quality control isolate of Candida krusei. Antimicrob Agents Chemother 2008;52:1500-2.

[12] Espinel-Ingroff A, Arthington-Skaggs B, Iqbal N, Ellis D, Pfaller MA, Messer S, et al. Multicenter evaluation of a new disk agar diffusion method for susceptibility testing of filamentous fungi with voriconazole, posaconazole, itraconazole, amphotericin B, and caspofungin. J Clin Microbiol 2007;45:1811-20.

[13] Rodriguez-Tudela JL, Alcazar-Fuoli L, Mellado E, Alastruey-Izquierdo A, Monzon A, Cuenca-Estrella M. Epidemiological cutoffs and cross-resistance to azole drugs in Aspergillus fumigatus. Antimicrob Agents Chemother 2008;52:2468-72.

[14] Schoeni MH. To treat or not to treat with antifungal agents? J Cyst Fibros $2005 ; 4: 213-4$. 
[15] Seidler MJ, Salvenmoser S, Müller FM. Aspergillus fumigatus forms biofilms with reduced antifungal drug susceptibility on bronchial epithelial cells. Antimicrob Agents Chemother 2008;52:4130-6. 


\section{Table 1}

Susceptibility to amphotericin B (AMB), caspofungin (CAS), itraconazole (ITC), voriconazole (VRC) and posaconazole (PSC) of 159 Aspergillus fumigatus isolates collected from the sputa of 11 cystic fibrosis patients

\begin{tabular}{llllll}
\hline Patient $(n)^{\text {a }}$ & AMB & CAS & ITC & VRC & PSC \\
\hline A (39) & $0.25-1$ & $\leq 0.03-0.25$ & $0.5-2$ & $0.25-1$ & $0.06-0.5$ \\
B (54) ${ }^{\text {b }}$ & $0.25-1$ & $\leq 0.03-0.25$ & $0.5-2$ & $0.25-1$ & $0.06-0.25$ \\
C (5) ${ }^{\text {b }}$ & $0.5-1$ & $0.06-0.125$ & $0.5-2$ & $0.25-0.5$ & 0.25 \\
D (14) ${ }^{\text {b }}$ & $0.5-1$ & $\leq 0.03-0.125$ & $0.5-2$ & $0.25-0.5$ & 0.125 \\
E (13) ${ }^{b}$ & $0.5-1$ & $\leq 0.03-0.06$ & $0.5-1$ & $0.25-0.5$ & $0.125-0.25$ \\
F (4) & $0.25-0.5$ & $0.125-0.25$ & $0.5-1$ & 0.5 & 0.125 \\
G(1) & 0.5 & 0.06 & 0.5 & 0.25 & 0.125 \\
H (15) & $0.5-2$ & $\leq 0.03-0.125$ & $0.5-1$ & $0.25-0.5$ & $0.06-0.25$ \\
I (10) & $0.25-1$ & $\leq 0.03-0.06$ & $1-2$ & 0.5 & $0.125-0.25$ \\
J (2) & $0.5-1$ & 0.06 & $0.5-1$ & 0.5 & $0.125-0.25$ \\
K (2) & 0.5 & $0.125-0.25$ & 0.5 & $0.25-0.5$ & 0.125 \\
\hline
\end{tabular}

\footnotetext{
${ }^{a}$ Number of isolates recovered from each patient in parenthesis.

${ }^{b}$ Patients B, C, D and E received antifungal azole therapy (details in Section 2.2).
} 